\title{
Chameleon: Next Generation Sample Preparation for CryoEM based on Spotiton
}

\author{
Michele C. Darrow ${ }^{1}$, John P. Moore ${ }^{1}$, R. John Walker ${ }^{1}$, Klaus Doering ${ }^{1}$ and Russell S. King ${ }^{1}$ \\ 1. TTP Labtech LTD., Melbourn Science Park, Hertfordshire, UK.
}

In the workflow for high-resolution structural determination, improvements in microscope stability, direct detectors and image processing have shifted the bottleneck to sample preparation. The process of obtaining a film of vitreous ice of an appropriate thickness, with evenly distributed particles is not straightforward. Many of the current vitrification methods are highly variable, necessitating the costly step of screening each grid in an electron microscope (EM). Additionally, relatively large sample volumes are required and then lost during the process of blotting, and further grid losses are sustained during the manual handling required to transfer frozen grids into storage, and through poor traceability of storage locations.

The chameleon system is a blot-free, pico-litre dispense instrument for quickly and robustly freezing samples for use in cryoEM (Figure 1A). The chameleon system is based on Spotiton [1,2] and uses selfwicking copper nanowire grids to form the thin sample film (Figure 1B) [3]. This process occurs 'on-thefly' as the grid passes in front of the dispenser on its way to the cryogen bowl, resulting in a stripe of sample across the frozen grid.

The user interacts with the system by loading in hydrophilic grids, sample, and cryogens. The grid is picked up by the on-board tweezers and the sample is aspirated by the dispenser. These components are then brought together in a high-humidity chamber for a high-speed plunge into liquid ethane. A short video is captured just before the grid with sample is plunged into the liquid ethane, allowing for automatic assessment of the grid condition and ice thickness just prior to freezing ( $\sim 50 \mathrm{~ms})$. Importantly, this assessment takes place without preparing or loading the grids into a cryoEM, reducing the costs associated with screening. Once the grid is frozen and has passed the on-board assessment, it is then placed into a storage button and the system automatically prepares for freezing the next grid. These steps remove the need for multiple manual grid handling steps, especially those which take place in cryogenic conditions.

The minimum sample required is $5 \mu$ l. This is pipetted into a sample block and kept at a user-specified temperature while awaiting aspiration. While it is possible to aspirate smaller volumes, to address the effects of sample mixing with the working fluid, the minimum recommended aspiration amount is $3 \mu 1$. While in traditional plunge freezing methods 3-5 $\mu 1$ of sample is enough to produce only one or two frozen grids, with chameleon this amount could produce hundreds of frozen grids.

Understanding the kinetics of proteins during thin-film formation and the effects of local environments such as the grid support and air-water interface are ongoing questions in the field of cryoEM. However, recent publications have discussed and demonstrated the negative impact on proteins during exposure to these environments [4,5] and a decrease in the time between sample dispense and grid freezing has been shown to reduce preferred orientation and aggregation for some samples [6]. In comparison to other freezing methods where the sample deposition alone or in conjunction with blotting can take several seconds, the chameleon system reduces this 'dispense-to-plunge' time to under $100 \mathrm{~ms}$.

The grid freezing process can sometimes be overwhelming due to the need to work efficiently to bring the cryogens, sample, and grids together in concert. Even for an experienced user, noting down details of the experiment while freezing can be difficult, and many times grids are not imaged immediately, 
compounding the issue. This can result in poor outcomes during data collection and an overall lack of sample traceability. To address this, we have included a reporting function within the chameleon software that produces a freezing report which includes sample, grid, instrument and environmental parameters, and an image of the grid just prior to freezing (Figure 1C). This allows the user to be fully engaged with the freezing process and able to manage decisions about grid retention safe in the knowledge that key sample details are automatically recorded.

Safety and robustness are key features of the chameleon system. Interlocks are used to keep both the user and the instrument safe and a motorized cryogen bowl prevents the sloshing of liquid nitrogen and liquid ethane. The addition of liquid nitrogen level sensing and user-specified ethane temperature control has reduced the volumes of each that are necessary and removed the need to regularly check the cryogen bowl.

The next steps in development of the chameleon system will be to include on-board glow discharge to create a full workflow from naïve grid to frozen grid with reduced manual grid handling. We will also explore new aspects of grid manufacturing to create grids with alternative materials and various patterned hole diameters and spacings.

Together with the improvements around manual handling of grids, and the automated set-up, assessment, and clean-up software workflows, the chameleon is a sample vitrification system with walk-up usability (Figure 1D) that also creates opportunities for cutting-edge research despite poorly behaved cryoEM samples.

\section{References:}

[1] I Razinkov et al., Journal of Structural Biology 195 (2016), p. 190.

[2] V Dandey et al., Journal of Structural Biology 202 (2018), p. 161.

[3] H Wei et al., Journal of Structural Biology 202 (2018), p. 170.

[4] R Glaeser et al., Biophysical Journal 110 (2016), p. 749.

[5] R Glaeser, Current Opinion in Colloid \& Interface Science 34 (2018), p. 1.

[6] A Noble et al., Nature Methods 15 (2018), p. 793.
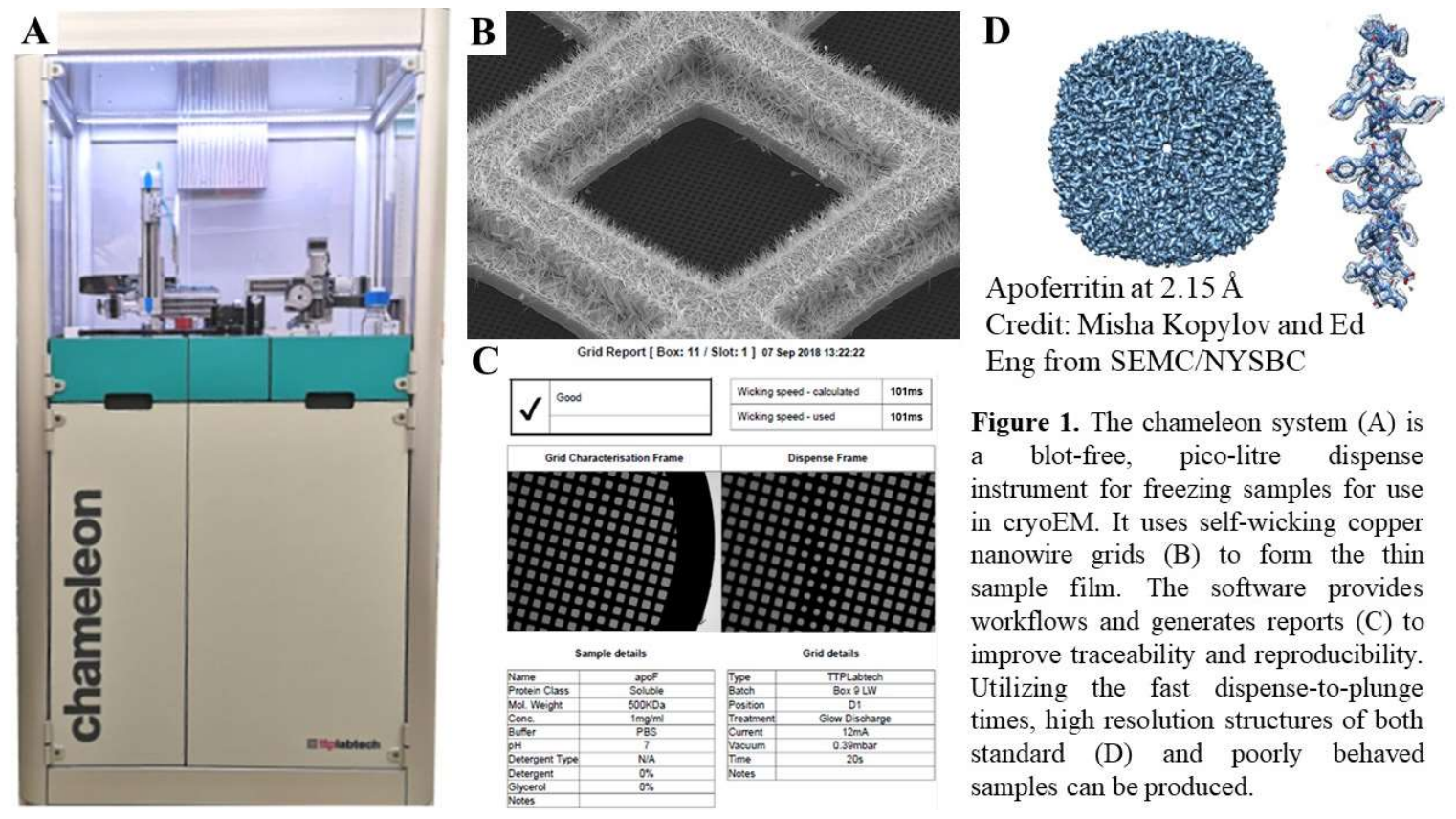

Figure 1. The chameleon system (A) is a blot-free, pico-litre dispense instrument for freezing samples for use in cryoEM. It uses self-wicking copper nanowire grids (B) to form the thin sample film. The software provides workflows and generates reports (C) to improve traceability and reproducibility. Utilizing the fast dispense-to-plunge times, high resolution structures of both standard (D) and poorly behaved samples can be produced. 